\section{Allergologie für die Ohren}

0 ffensichtlich werden Hörbücher immer populärer. So gibt es jetzt auch ein Hörbuch über allergische Krankheiten für interessierte Laien. Die Autoren Fuchs, Bauer und Lämmel sind auf der $\mathrm{CD}$ zu hören, ergänzt durch den professionellen Sprecher Tschernek. Sie alle machen ihre Sache sehr gut, haben angenehme Stimmen und so hört man gerne zu. Die Zwischenmusik von O.P. Graber ist dagegen eher etwas langweilig - zumindest weit entfernt von Ennio Morricone.

Der Text ist durchgehend sehr gut verständlich und für betroffene oder nur wissensdurstige Hörer hilfreich, richtig und wegweisend. In 60 Minuten werden kapitelweise wichtige allergische Krankheiten dargestellt, mit zeitlichen Unterschieden, in etwa gewichtet nach der Bedeutung der Krankheitsbilder. Es werden besprochen: Heuschnupfen, Kontaktallergie, Bienen- und Wespengiftallergie, Lebensmittelallergien, Unverträglichkeiten oder Pseudoallergien, Allergien bei Kindern sowie Heuschnupfen und Asthma bei Kindern.

Welche Wünsche könnten beim Patienten offen bleiben? Möglicherweise war es nachteilig, dass weder ein HNO-Arzt noch ein Lungenfacharzt eingeschaltet wurde. So ist das Thema allergisches und berufliches Asthma beim Erwachsenen deutlich unterrepräsentiert. Es fehlen auch nähere Erläuterungen $\mathrm{zu}$ den komplexen Erkrankungen der oberen Luftwege, deren Differenzialdiagnose und Therapiemöglichkeiten, über Antihistaminika und topische Glukokortikoide hinaus.

Das Thema Nahrungsmittelallergien und -intoleranzen wird sehr breit abgehandelt. Andererseits wird auf diese Weise genügend Zeit für die komplexe Materie eingeräumt. Typische Kreuzallergien sind richtig dargestellt. Die notwendige Kritik gegenüber den Testmöglichkeiten ist enthalten. Sehr gut ist die Darstellung der Intoleranzreaktionen für den Laien, vor allem auch die Unterscheidung von Allergie, Pseudoallergien und Enzymdefekten. Lebensmittelempfehlungen werden dagegen zeitlich überbetont. Vielleicht hilft dies, die häufig von Patienten überschätzte Bedeutung von Nahrungsmittelallergien gegenüber anderen Ursachen von Magen-Darm-Beschwerden richtig zu stellen.

$\mathrm{Zu}$ den besten Kapiteln gehört die Darstellung von Allergien bei Kindern. Allerdings wird möglicherweise in weiteren Auflagen die Bedeutung der frühkindlichen Ernährung für die Allergieprävention revidiert werden müssen, folgt man neueren Studien zu diesem
Thema. Ausgezeichnet ist die ausgewogene Darstellung des aktuellen Standes der spezifischen Immuntherapie, insbesondere bezüglich des Vergleichs von subkutaner und sublingualer Therapie. Die Bedeutung der komplementären bzw. nicht schulmedizinischen Verfahren wird angemessen dargestellt. Besonders erfreulich ist die häufige Botschaft: "Suchen Sie Ihren Allergologen auf!“

Das Werk ist gelungen und kann allen Interessierten empfohlen werden. Insbesondere kann das Hörbuch eine große Hilfe auch für uns Ärzte sein, da hier zahlreiche Fragen beantwortet werden, die kaum so ausführlich in der Sprechstunde besprochen werden können. Keine Frage aber: Auch nach dem Anhören der CD wird der Patient weitere Fragen haben - vielleicht sogar mehr als zuvor, da er nun besser und auf hohem Niveau informiert ist. Wie man aber weiß, ist gehört nicht unbedingt auch verstanden ...

Prof. Dr. Gerhard Schultze-Werninghaus,

Bochum

Bauer C.P., Fuchs T., Lämmel S.

Allergien verstehen, behandeln, vorbeu-

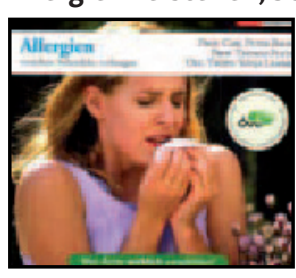
gen (Audio-CD) Etsdorf/Kamp: Galila-Hörbuchverlag, 2007 $14,95 €$ ISBN 3-902533०8-0

\title{
Guter Einstieg in die Allergologie
}

D er bei Allergologen recht bekannte Fachverlag Dustri hat mit diesem Buch eine erste „Hilfestellung" für Einsteiger in Diagnostik und Therapie der Allergien vorgelegt. Das Buch ist recht übersichtlich gehalten, allerdings sparsam illustriert. Besonders bei methodischen Fragen und im Kapitel „Laboruntersuchungen" kann es punkten. Dagegen werden die Grundlagen der Sensibilisierung und der allergischen Reaktionsweisen nur knapp behandelt. Die wichtigsten allergologischen Krankheitsbilder werden zwar auch mit klinischen Bildern und ihrer In-vivo-Diagnostik umrissen, mögliche Differenzialdiagnosen und das breite Spektrum des therapeutischen Vorgehens kommen allerdings etwas zu kurz, wie z. B. auch die Überschneidungen allergischer und nicht allergischer Unverträglichkeitsreaktionen kaum erwähnt werden.

Die symptomatische medikamentöse und die spezifische Immuntherapie sowie das Vorgehen bei allergologischen Notfällen sind für den Anfänger ausreichend erörtert. Jedoch könnte das Buch für die weitere Vertiefung beispielsweise Literaturhinweise und Internetadressen bereithalten. Das Werk bietet für Ein- steiger und v.a. auch für an labormethodischen Fragen Interessierte aktuelle Informationen. Es gibt aber die Breite der praktisch-klinischen Allergologie nicht so umfassend wieder, wie man es sich gerade auch für den zweiten Blick eines Anfängers wünscht.

Dr. Ulrich Mutschler, Hildesheim

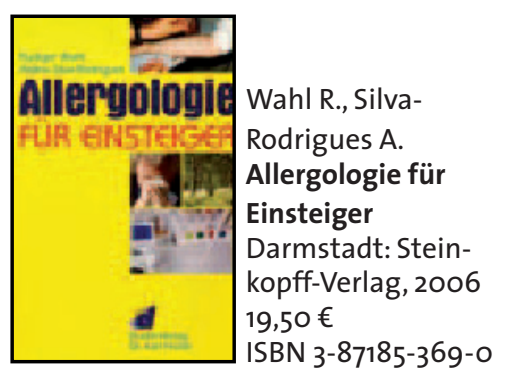

